Conclusions Modified Kobayashi score performed best among the established international risk scores for IVIG resistance with good specificity but fair sensitivity in our population. We have designed a preliminary novel clinical risk score for IVIG resistance, $H K-K D$ score $v 1$, with sufficient sensitivity and specificity for potential clinical use.

\section{A NOVEL TWO-TIERED DEVELOPMENTAL SCREENING PROGRAMME FOR SINGAPOREAN TODDLERS: A QUALITY IMPROVEMENT REPORT}

Clara Nwe Nwe Linn Oo, Yoke Hwee Chan, David Chee Chin Ng, Truls Ostbye, Pratibha Keshav Agarwal, Sita Padmini Yeleswarapu. Singapore

\subsection{6/bmjpo-2021-RCPCH.127}

Background Early identification of developmental delays with timely intervention, especially before the age of 3 years, can optimise child development. With the pre-existing model of developmental surveillance in Singapore however, children with suspected developmental delays first consult a developmental specialist at a median age of 44 months to undergo a detailed developmental assessment and diagnosis, and only then receive intervention.

Objectives Our Quality Improvement team agreed that improvements made to the pre-existing model of developmental surveillance would enable earlier identification of developmental delays thereby triggering early intervention. Hence, we aimed to increase the proportion of children seen in primary care who screen positive for potential developmental delays by $5 \%$ before the age of 3 years, with the use of a novel twotiered developmental screening programme in a primary care setting, without increasing the false positive rates.

Methods We describe the implementation and evaluation of a novel two-tiered developmental screening programme into the existing 9-month and 18-month screening schedule, with an additional screening at 30 months to replace the pre-existing 36-month screening of the National Child Health Surveillance Programme. The new programme utilizes some of the American Academy of Pediatrics's recommended screening tools: Parents' Evaluation of Developmental Status (PEDS), Parents' Evaluation of Developmental Status - Developmental Milestones (PEDS-DM) and The Ages \& Stages Questionnaires (ASQ) as part of our new two-tiered screening programme. We evaluated this programme using quality improvement methods by measuring the proportion of children referred to child development units after positive screening for developmental delays under the new programme, with a pre-post and with-without comparison. We also studied risk factors associated with positive screening in the programme.

Results The two-tiered screening was most effective at 18 months with the proportion referred improving from $3.5 \%$ to $7.1 \%$ over a 6 -month period. In addition, the referred proportion at 18 months was also significantly higher at the primary care center where the two-tiered screening was implemented as compared to other primary care centres in the same healthcare cluster who were utilizing the pre-existing screening (7.8\% compared to $3.2 \%)$. For those who received further assessment by developmental specialists after the two-tiered screening, $100 \%$ received a definitive diagnosis of developmental delays, similar to our baseline data. The risk factors identified for screening positive among Singaporean toddlers: male gender, low maternal education, preterm births and low income shed light on population subgroups which may require more attention and resources in future.

Conclusions Our quality improvement efforts have facilitated the integration of a novel two-tiered screening programme into the pre-existing screening schedule, with improved referral proportions without increasing the screening process's false positive rates. While we highlight challenges in implementation that need to be addressed, our findings support a potential nation-wide adoption of the two-tiered programme.

\section{BEHAVIORAL ANALYSIS OF DRUG NON-COMPLIANCE IN PEDIATRIC EPILEPSY}

Fatima Mazahir, Fatima Mazahir. United Arab Emirates

\subsection{6/bmjpo-2021-RCPCH.128}

Background Epilepsy ranks among the commonest chronic neurological diseases, with a global estimate of 5 million people diagnosed annually. It is broadly defined as having two or more unprovoked seizures linked to a spectrum of behavioral, psychiatric, and cognitive disorders resulting from birth injuries, endemic conditions, or traumatic brain injuries. Worldwide, up to 50 million people are affected, of whom 10.5 million are children below 15 years of age. Despite its high burden, up to $70 \%$ of epileptic patients could live seizure-free with accurate and timely diagnosis and appropriate long-term treatment with relatively cheap and effective anti-epileptic drugs (AEDs). The main obstacle in achieving this goal is the medication non-compliance more frequently reported in middle-income (44.4\%) and low-income countries (74.8\%). Adherence relates to using the prescribed treatment appropriately with regards to time, quantity, period, and manner.

Objectives We aim to understand the behaviors underlying non-compliance to AEDs in children from marginalized communities to formulate specific interventions to improve the treatment gap.

Methods The target population was children diagnosed with epilepsy under 15 years from lower- and middle-income communities (LMIC), and non-adherence to AEDs was identified as the target behavior. A literature review with keywords 'Pediatric Epilepsy,' 'LMIC,' 'Non-Compliance,' 'Medication Adherence,' and 'Behaviors' was conducted via Google Scholar and PubMed. Based on the abstract review, 22 articles were shortlisted for full article review, out of which 7 articles were finalized for analysis. The primary factors identified were fear, stigma, concerns about the medication's safety, and insufficient knowledge of the disease. These behaviors were analyzed in detail via the COM-B Model of Capability, Opportunity, and Motivation proposed by Miche et al. This analysis provides a framework to link targeted interventions to the specified behaviors and policy domains to the outlined interventions via the Behavior Change Wheel (BCW) Methodology.

Results A wide range of physical, psychological, and social factors underpinning the non-compliance to the AEDs were identified and mapped to the COM-B Model (table 1) of psychological or physical capabilities, social and physical opportunities, and automatic and reflective motivation. Forgetfulness was the primary issue at around $54.25 \%$, followed by disapproved perceptions about epilepsy such as denial, selfpity, social exclusion, and fear of societal exclusion $(20.55 \%)$; and mistrusting their physician leading to increased concerns about medication safety and side-effects (8.87\%). 


\begin{tabular}{|c|c|c|}
\hline Capability & Opportunity & Motivation \\
\hline Psychological & Social & Reflective \\
\hline - Parent & - Parent & - Parent \\
\hline o Cognitive Function & oStigma & o Negative Attitudes \\
\hline \multirow[t]{3}{*}{ o Education Level } & ○ Religious/cultural beliefs & o Treatment Concerns \\
\hline & olncome & ○ Alternative Therapies \\
\hline & & - Outcome Expectations \\
\hline Physical & Physical & Automatic \\
\hline - Child & - Both & - $\underline{\text { Both }}$ \\
\hline oDrug Ingestion & o Interrupted access to health care facilities & ○ Fear and Anxiety \\
\hline o Parent & o Inconsistent doctor-patient relationship & \\
\hline o Forgetfulness & - Cost of medication & \\
\hline
\end{tabular}

Conclusions This study's main application lies in formulating interventions related to acknowledging misconceptions and stigmas related to Epilepsy and improving health-care systems by creating a team-based approach to patient care in meeting the required demands. These interventions may also be linked to devise applicable policies to impact reforming target behavior significantly.

\section{GENOTYPE/PHENOTYPE CORRELATIONS IN 125 CHINESE PATIENTS WITH TUBEROUS SCLEROSIS: A 29 YEARS' EXPERIENCE IN HONG KONG}

Samuel Yan Lik NG, Ivan FM Lo, Ho-Ming Luk. Hong Kong

\subsection{6/bmjpo-2021-RCPCH.129}

Background Tuberous Sclerosis Complex (TSC) is a multisystemic neurocutaneous disorder of autosomal dominant inheritance, with characteristic presentation of benign hamartomatous lesions across the brain, kidney, heart, eyes, skin and lungs.

Objectives To study the mutation spectrum and phenotypic characteristics of TSC patients in the Chinese population, as well as to delineate the underlying genotype-phenotype correlations and compare with previous studies.

Methods 120 patients suspected of TSC were referred to the Clinical Genetic Service (CGS), Department of Health, HKSAR for evaluation between 9/1991 and 8/2020. Blood samples from 105 patients presented with $\geq 1$ major/ $\geq 2$ minor diagnostic features according to the latest diagnostic criteria $[1,2]$ are taken. Comprehensive genetic testing including sequencing and Multi-ligand probe-dependent Amplification (MLPA) was performed. Family screening was performed on molecularly confirmed cases. In total, 133 patients had a definite diagnosis, in which the 125 Chinese patients are included into the study. Statistical analyses (Pearson $\chi 2$ tests, Fisher exact test and ANOVA) are performed using SPSS version 26.0.

Results Pathogenic genetic alternations are identified in $72.0 \%$ patients (90/125), in which $26.4 \%$ (33/125) have TSC1 and $45.6 \%(57 / 125)$ have TSC2 mutations. 28 novel mutations are reported while familial cases account for 23.2\% (29/125). Males have significantly more subependymal nodules (47/55 vs $34 / 50 ; p=0.033)$ than females, whereas de novo cases have more cortical tubers $(69 / 82$ vs $14 / 24 ; p=0.007)$ and renal angiomyolipoma $(44 / 85$ vs $3 / 21 ; p=0.002)$ than familial cases.
TSC2 cases have more frequent mental retardation (29/42 vs $4 / 25 ; \quad \mathrm{p}<0.001)$, cardiac rhabdomyoma $(21 / 45$ vs $3 / 25$; $\mathrm{p}=0.003)$, renal angiomyolipoma $(24 / 49$ vs $5 / 27 ; \mathrm{p}=0.009)$ and facial angiofibromas $(37 / 50$ vs $16 / 31 ; p=0.039)$ than TSC1, while mutation-negative cases show less subependymal nodules $(14 / 23$ vs $65 / 77 ; p=0.015)$ than mutation-positive cases. Similarly, TSC2 cases show higher occurrence of subependymal nodules $(44 / 50$ vs $14 / 23 ; \mathrm{p}=0.012)$ than mutationnegative cases though mutation-negative patients have more frequent mental retardation $(10 / 21$ vs $4 / 25 ; \mathrm{p}=0.020)$, autism spectrum disorder $(4 / 18$ vs $0 / 23 ; \mathrm{p}=0.030)$ and renal angiomyolipoma $(12 / 23$ vs $5 / 27 ; \mathrm{p}=0.012)$ than TSC1 cases. There are no significant phenotypic differences between patients with missense and protein-truncating mutations in TSC2, while TSC2 missense mutations are associated with more mental retardation $(17 / 21$ vs $4 / 23 ; \mathrm{p}<0.001)$, cardiac rhabdomyoma $(11 / 21$ vs $2 / 24 ; p=0.001)$, renal angiomyolipoma (13/23 vs $4 /$ $24 ; p=0.004)$ and renal cysts $(6 / 23$ vs $1 / 24 ; p=0.048)$ than those with TSC1 protein-truncating mutations. Mutations in the coiled-coil region (TSC1) are significantly associated with nail abnormalities. All 14 antenatal-onset patients have cardiac rhabdomyoma. Meanwhile, they have less seizures (6/11 vs $55 / 64 ; p=0.027)$ than paediatric-onset cases but have higher frequencies of mental retardation $(5 / 11$ vs $0 / 11 ; \mathrm{p}=0.035)$ than adult-onset patients. Generally, paediatric-onset patients have more neurological manifestations, while initial presentations of adult-onset TSC are more diverse. The predictive yield of antenatal cardiac rhabdomyoma on TSC is $63.6 \%$.

Conclusions The overall phenotypic spectrum and genotypephenotype correlations in our Chinese cohort are compatible with literature. 28 novel mutations have been reported in this study.

\section{A SURVEY OF PARENTAL KNOWLEDGE, ATTITUDE AND PRACTICE OF USING CHILD CAR SEAT RESTRAINTS IN SINGAPORE, WITH A SYSTEMATIC REVIEW}

Xi Yun Ling, Alvin Jia-Hao Ngeow, Mary Grace Sy Tan, Daisy Kwai-Lin Chan. Singapore

\subsection{6/bmjpo-2021-RCPCH.130}

Background Motor vehicle accidents are associated with significant mortality and morbidity in adults and children. The annual number of accidents with injury in Singapore remained at over 7600 for the past 5 years. Child car restraints prevent 